\title{
Building capacity in primary care: the implementation of a novel 'Pharmacy First' scheme for the management of UTI, impetigo and COPD exacerbation
}

\author{
Fiona Stewart ${ }^{1}$, Gail Caldwell ${ }^{2}$, Kirstin Cassells ${ }^{3}$, Jonathan Burton ${ }^{4}$ and Anne Watson ${ }^{5}$ \\ ${ }^{1}$ Associate Postgraduate Pharmacy Dean, NHS Education for Scotland, Formerly Lead Pharmacist (Primary and \\ Community Care), NHS Forth Valley, Scotland \\ ${ }^{2}$ Director of Pharmacy, NHS Ayrshire and Arran, Formerly Director of Pharmacy, NHS Forth Valley, Scotland \\ ${ }^{3}$ Community Pharmacy Development Pharmacist, NHS Forth Valley, Formerly Community Pharmacy Champion, NHS \\ Forth Valley, Scotland \\ ${ }^{4}$ Community Pharmacist, Community Pharmacy Forth Valley, Scotland \\ ${ }^{5}$ Professor, Postgraduate Pharmacy Dean, NHS Education for Scotland, Scotland
}

\begin{abstract}
Aim: This service aimed to improve patient access to treatment for urinary tract infections (UTI), impetigo and exacerbation of chronic obstructive pulmonary disease (COPD) and relieve pressure on general practice and out of hours services. Background: In 2016, a service (Pharmacy First) was introduced in Forth Valley for the management of UTI, impetigo and exacerbation of COPD using patient group directions in community pharmacies. Trained pharmacists supplied a limited range of prescription medicines. Pathways for GP referral were defined. After 5 months of implementation, the service was evaluated. Methods: A quantitative evaluation was undertaken. Feedback was sought from patients, GPs, pharmacists and GP reception staff, using structured questionnaires. Pharmacy records were used to assess referrals and pharmacy data summarised the number and type of consultations. Basic cost data was obtained from the Health Board. Findings: In all, 75 pharmacies (of 76), and all 55 GP practices in the area, participated in the service. Over a 5-month period, 1189 cases were managed, the majority being for UTI (75.4\%) followed by impetigo (15.2\%), then COPD $(9.3 \%)$. Of all cases, $77.9 \%$ were prescribed medication by the pharmacist, $9.1 \%$ were given advice only and $16.7 \%$ were referred to the GP. Independent clinical assessment of a random sample of $30 \mathrm{GP}$ referrals considered all to be 'appropriate'. Feedback was received from 69 pharmacists, $34 \mathrm{GPs}, 54$ reception staff and 73 patients. Patients were very satisfied with the service, most frequently citing the 'quick and efficient' access to treatment, and a 'professional service'. Two thirds of GPs $(67 \%)$ and $59 \%$ of reception staff found the service useful, mainly because it reduced pressure on GP appointments. A further cost benefit evaluation would allow objective assessment of the value of this service.
\end{abstract}

Key words: community pharmacy; service delivery; quantitative evaluation; acute minor infection; patient satisfaction

Received 25 April 2017; revised 19 September 2017; accepted 8 December 2017;

first published online 24 January 2018

Correspondence to: Fiona Stewart, Associate Postgraduate Dean, NHS Education for Scotland, Formerly NHS Forth Valley, 3rd Floor, 2 Central Quay 89 Hydepark Street, Glasgow G3 8BW, Scotland. Email: fiona.stewart@nes.scot.nhs.uk

(C) Cambridge University Press 2018

\section{Introduction}

The challenges facing the General Practice workforce are well known. The latest workforce survey in Scotland found a $2 \%$ reduction in the number of 
whole time equivalents of General Practitioners (GPs) and over a third of GPs were over 50 years (ISD, 2016). Across the United Kingdom there is a predicted shortfall in the number of GPs over the next decade (Smith et al., 2013). In both England and Scotland there are more young women going into general practice and often those taking on roles are choosing to work a reduced number of hours so more GPs may need to be recruited to maintain the workforce (CWFI, 2014). To address these challenges, novel models of service redesign are being considered and tested. Making use of the pharmacy workforce has been acknowledged and the Royal Pharmaceutical Society recommended that 'Local Commissioners include pharmacist expertise in all care pathways that use medicines including the formal involvement of community pharmacists' (RPSGB, 2015). Furthermore, it has generally been acknowledged that GPs and pharmacists should work more closely for patient benefit and facilitating self-care is an area of initial focus (Joint Statement RPS (Scotland) RCGP (Scotland), 2011).

The UK concept of 'Pharmacy First' describes clinical services in which patients are encouraged to seek advice and treatment from community pharmacists before making an appointment in general practice. In England pharmacy first services are generally used for management of minor ailments. In Scotland the term has been used slightly differently and the service is distinct from the Minor Ailment Scheme (MAS). In Scotland it has been used for patients to seek treatment for common clinical conditions that would generally require a GP appointment to obtain a prescription only medicine.

This model of 'Pharmacy First' used patient group directions (PGDs) as the legal framework for supplying appropriate prescription medicines. Legislation establishing PGDs was introduced in 2000. The current legislation for PGDs is included in The Human Medicines Regulations 2012 (Legislation. gov.uk, 2012). PGDs allow some registered health professionals to supply and/or administer a specified medicine(s) to a pre-defined group of patients, without them having to see a doctor. NICE guidance states that supplying or administering medicines under PGDs should only be in situations that are an advantage for patient care and do not compromise patient safety (NICE, 2013).

\section{The Pharmacy First Service in Forth Valley}

The Pharmacy First initiative has been in operation in Forth Valley since March 2016 with the aim of improving patient access to treatment for uncomplicated urinary tract infections (UTI), impetigo and exacerbation of chronic obstructive pulmonary disease (COPD). Patients are managed in community pharmacy by trained pharmacists and receive either treatment, advice or referral. The service also aimed to increase access to rescue antibiotics and steroids for patients with an exacerbation of COPD with hand-held, selfmanagement record cards.

Training was provided for pharmacists via three protected learning sessions which provided Pharmacy First packs containing all the documentation. There was a presentation on antimicrobial stewardship, and treatment of exacerbation of COPD, workshops on provision and use of trimethoprim and fusidic acid PGDs and explanation of the out-of-hours referral pathway. In addition pharmacists had to complete a mandatory UTI education module. Pharmacists who could not attend an event or had not had an update on antimicrobial stewardship in the last 12 months were also asked to complete an Antimicrobial and Infection Management training module.

A secondary aim of the service was to free up GP and out of hours services (OOHs) appointments for more complicated clinical cases and emergency treatment. The service is available from local community pharmacies both within GP opening hours and out of hours. There are 76 community pharmacies in NHS Forth Valley and all but one signed up to the service. A total of 12 that could not attend initial training were brought on board later. GP practices were sent information directly and also via their practice managers on two occasions. In addition practice pharmacists were asked to raise awareness of the service at practice meetings.

A comprehensive communication strategy was employed by NHS Forth Valley to ensure the public and health professionals were informed and aware of this new service. Posters were sent to all community pharmacies, GP practices, OOHs, accident and emergency and minor injuries departments in the area. Facebook and twitter adverts were used and the service was highlighted in local newspapers and press releases. Information regarding inclusion/exclusion criteria were sent to all GP practice managers and practice pharmacy staff. Power-point presentations were created and sent to all GP practice managers to run on TV screens in waiting rooms.

The implementation of the service was overseen by the Pharmacy Services team of NHS Forth Valley 
and led by the Community Pharmacy Development team. Pharmacists are remunerated from NHS Forth Valley. There was an initial start-up fee of $£ 100$ and payment thereafter was dependent on the return of claims forms which document the number of cases and the action taken. Fees were provided per consultation regardless of whether it resulted in supply, referral or advice only.

There are many initiatives addressing the challenges in GP and utilising the pharmacist workforce in many different models. However, the academic literature does not yet reflect this and evaluations of these initiatives can be limited in scope and difficult to identify. A search of the academic literature identified just one study of the use of PGDs in community pharmacy for the supply of emergency hormonal contraception (Bissell et al., 2006). There is a wider literature available about pharmacist prescribing which is of some relevance although pharmacists require additional qualifications to become prescribers. A review of patient and public views and experiences of pharmacist prescribing (Famiyeh and McCarthy, 2017) found public support in limited situations such as minor ailments, and chronic conditions. Patients and the wider public noted concerns about safety arising from lack of pharmacists' access to medical records.

This paper presents a pilot evaluation of the first 5 months of service implementation using a PGD model. The aims of this evaluation was to evaluate the initiative from the perspective of patients, GP practices (GPs and reception staff) and pharmacists, to determine whether the referral system was used appropriately and to review the clinical areas covered. Although it is most relevant to a UK audience it is hoped that general lessons of shifting care from general practice to pharmacy will have wider interest beyond the United Kingdom.

\section{Methods}

\section{Study design}

A quantitative, descriptive evaluation has been undertaken. Structured questionnaires were used with pharmacists, patients, GPs and GP reception staff. Patient referral data was held on pharmacy records and pharmacists submitted forms detailing cases to NHS Forth Valley, for payment. These were used to record the number and type of cases and the action taken.
Data collection tools (questionnaires) were developed by Forth Valley Community Pharmacy Development team with advice from the local Quality Improvement team. Previous patient feedback questionnaires and GP questionnaires were used to inform data collection questionnaires. Three Community Pharmacy Champions tested the questionnaire. The questionnaires used a mixture of closed and open format questions.

\section{Data collection}

Data collection was undertaken by three Pharmacy Champions and the Pharmacy Project Support Manager. Feedback questionnaires were either administered face to face, or, if the GP/pharmacist was unavailable, the questionnaire was left for self-completion. Telephone and face to face administered, structured interviews were used particularly with GP reception staff. This pragmatic, mixed approach was considered to be the most time efficient method of maximising participation.

Regarding appropriateness of referral data, a random one in four sample of 30 cases was selected (using random number tables) for review by a clinician (from 124 cases that had been referred by mid July). The clinician reviewed the reason for referral and noted whether it was clinically appropriate and if not, why not. The clinician was a senior GP partner with 10 years of experience in primary care. This clinician was approached to undertake this task as he was a designated medical practitioner for pharmacists undertaking the independent prescribing qualification. Therefore, this clinician was aware of the role of prescribing pharmacists.

\section{Data management and analysis}

Data were entered into Microsoft Excel. Separate worksheets were created for each data set. Coded responses were used for categorical questions with free text responses for open questions. Verbatim responses were typed into a spreadsheet. No individual names were included in the data set to maintain anonymity and protect confidentiality. Analysis was undertaken in Excel with simple frequencies used to describe categorical data. Open question responses were analysed using content analysis in which common responses were grouped under themes, then counted. Verbatim text/quotes were used to illustrate themes. Analysis was undertaken by an independent researcher consultant, not linked to the NHS area. 


\section{Results}

\section{Pharmacy involvement}

A total of 75 pharmacies (of 76) were recruited to provide the pharmacy first service. Feedback data were available for 69 pharmacists. In terms of opening hours and the availability of the service, the majority were able to provide a service for at least 5 days with 21 providing a 6-day service and three providing a 7 -day service.

\section{Number and type of cases managed}

From March to end of July 2016, 1189 cases were managed under the Pharmacy First scheme. The number of cases per month were: 184 in March, 208 in April, 310 in May, 254 in June, 233 in July. The majority of cases were for UTI $(n=897$, $75.4 \%)$ with $9.3 \%(n=111)$ for COPD and $15.2 \%$ $(n=181)$ for impetigo. The majority $(77.9 \%)$ were prescribed a medicine via a PGD $(n=926)$ with $9.1 \% \quad(n=108)$ given advice only and $16.7 \%$ $(n=155)$ referred to the GP or OOHs (see Table 1). Those presenting with UTI were more likely to be referred to the $\mathrm{GP} / \mathrm{OOHs}$ than those with impetigo or COPD (15.5\% compared with $7.7 \%$ and $4.5 \%$, respectively).

\section{Referrals}

Reasons for referral are displayed in Table 2 under clinical condition. This data was obtained from pharmacy feedback questionnaires so was not provided for all 155 cases individually. Most referrals for UTI were related to being out with the age range. Generally this was because the patient was over 65 years but this was not always specified. Recent, previous treatment was also a common referral reason. For impetigo there were fewer cases but uncertainty about the clinical presentation was most frequently noted. For COPD there was a different pattern and many pharmacists noted that patients did not have the required hand-held card from the GP practice.

Of the original 30 random cases identified only eight could be used for review. In all, 10 cases had ticked the exclusion box but did not have a reason for referral documented (this problem became apparent during the project and the form was subsequently revised). Nine did not give any reason and in three cases, the pharmacy records could not be accessed as a locum was present. These cases were replaced by further random sampling until 30 cases were obtained for review. In all of these 30 cases, the referral was considered appropriate as defined by the objective clinical assessment.

\section{Service costs}

For this 5-month period, the cost to NHS Forth Valley for pharmacy fees was $£ 29615$. This was made up of:

1. Start-up fees: $£ 7500$ ( $£ 100$ per contractor).

Table 1 Description of cases (March to end July 2016)

\begin{tabular}{lrr}
\hline & Numbers of cases & \% of total \\
\hline Urinary tract infection & & \\
No. given advice & 139 & \\
No. of contacts with GP or OOHs & 682 & 75.4 \\
Medicine prescribed via PGD & 897 & \\
Total UTI & 5 & \\
COPD & 5 & \\
No. given advice & 101 & \\
No. of contacts with GP or OOHs & 111 & \\
Medicine prescribed via PGD & & \\
Total COPD & 27 & \\
Impetigo & 11 & \\
No. given advice & 143 & \\
No. of contacts with GP or OOHs & 181 & \\
Medicine prescribed via PGD & 1189 & \\
Total impetigo & & \\
All cases total & & \\
\hline
\end{tabular}

$\mathrm{OOHs}=$ out of hours services; PGDs = patient group directions; $\mathrm{UTI}=$ urinary tract infections; $C O P D=$ chronic obstructive pulmonary disease. 
Table 2 Reason for referral

\begin{tabular}{lr}
\hline & $n$ \\
\hline UTI referrals & 38 \\
Age & 17 \\
Recent previous treatment & 5 \\
Male & 4 \\
Pain symptoms & 4 \\
From out with Forth Valley area & \\
Other reasons: complicated symptoms, bleeding, recent surgery, possible & 3 \\
$\quad$ pregnancy, possible STD, discharge, systematically unwell & 2 \\
Impetigo & 2 \\
Unsure if Impetigo & 2 \\
Too young & \\
Multisite & 2 \\
Othecurrent symptoms & 2 \\
COPD & 2 \\
GP not supporting/not providing hand-held cards & \\
Recent antibiotic & \\
Recent treatment & \\
\hline
\end{tabular}

2. Protected learning time payment for up to two pharmacists per contractor code - (included payments for locums attending): $£ 11125$.

3. Consultation, advice, referral or supply fees: $£ 11890$.

Thus the ongoing annual costs of the service was estimated to be $£ 28536$ (discounting start-up fees and initial training). Additional locum training costs may be required (not provided in this pilot phase).

\section{Patient feedback}

Patient feedback questionnaires were distributed in April 2016 until 23 July 2016. In this time period, 73 responses were received. It is not possible to give an accurate baseline of how many were distributed. However, this represents $~ 9 \%$ of all patients seen under the scheme during this time period $(n=797$ for May-July). Of those who responded, 61 sought treatment for UTI, nine for impetigo and three for COPD exacerbation. Respondents were asked where they heard about Pharmacy First. Responses are displayed in Table 3.

Respondents were asked why they choose to go to community pharmacy rather than GP. Content analysis of free text responses identified the following reasons:

the GP/GP staff suggested it $(n=19)$

easier/more convenient $(n=18)$

quicker to access treatment/ saved time $(n=10)$ it was the weekend and the surgery was closed $(n=10)$

they could not get a GP appointment $(n=7)$

they received advice from their pharmacy $(n=5)$ other reason e.g. COPD self-management plan $(n=4)$

When asked how much do you agree or disagree with the following statement: 'I was happy with my experience of the Pharmacy First Service' all respondents either strongly agreed $(n=58)$ or agreed $(n=15)$. Respondents were asked to write a few words about their experience to justify their response. Content analysis identified five themes. These are display below with direct comments, as written, given as examples under each theme.

\section{Quick and efficient service $(\mathrm{n}=29)$}

'Following conversation with staff, I was seen in a timely manner. The screening did not take long at all, then I was swiftly given my prescription'

\section{Professional service $(\mathrm{n}=21)$}

Within this theme many respondents used the term professional directly: 'Prompt professional service'. Others described professional attributes such as discretion 'Private and discreet, good information'. Some referred to good consultation skills 'I felt well informed, listened to and at ease with the pharmacist carrying out the consultation'. 
Table 3 Patient awareness and experience

Where did you hear about pharmacy first? $(n=73)$

\begin{tabular}{|c|c|c|c|}
\hline & $n(\%)$ & & \\
\hline \multirow{7}{*}{$\begin{array}{l}\text { From GP } \\
\text { From GP staff } \\
\text { Newspaper } \\
\text { Pharmacist } \\
\text { Someone who had used the service } \\
\text { Other }\end{array}$} & $26(35.6)$ & & \\
\hline & $23(31.5)$ & & \\
\hline & $2(2.7)$ & & \\
\hline & $22(30.1)$ & & \\
\hline & $5(6.8)$ & & \\
\hline & 0 & & \\
\hline & $\begin{array}{l}\text { Yes } \\
n(\%)\end{array}$ & $\begin{array}{c}\text { No } \\
n(\%)\end{array}$ & $\begin{array}{c}\text { Missing } \\
n(\%)\end{array}$ \\
\hline Did you have any problems accessing the service? & $1(1.3)$ & $64(87.6)$ & $8(10.9)$ \\
\hline Would you recommend the pharmacy first service? & $64(87.6)$ & 0 & $9(12.3)$ \\
\hline Would you like to see other conditions treated by your community pharmacist? & $57(78.1)$ & $3(4.1)$ & $13(17.8)$ \\
\hline
\end{tabular}

One person had problems accessing the service and the reason given was that a locum pharmacist was working that day.

Helpful staff/pharmacists $(\mathrm{n}=18)$

'Was quick and easy service, helpful and would use again if required' and 'Very friendly staff and helpful giving advice'.

\section{Friendly staff/pharmacists $(\mathrm{n}=14)$}

'Very friendly and helpful. Did not have to wait to be seen and would definitely recommend this service to others. Was made to feel very comfortable when discussing the matter'.

\section{Informative service $(\mathrm{n}=10)$}

Some respondents emphasised the importance of getting good advice 'All about the expert advice I was given'. Others considered a knowledgeable and informative services as one of several features such as being quick and helpful 'Really quick system, the pharmacist was really helpful and knowledgeable, very happy with this service'.

\section{Convenient $(\mathrm{n}=2)$}

'Convenient, private and the service was very good'.

\section{Pharmacist feedback}

Sixty-nine of 75 participating pharmacies provided feedback (92\% response rate). The majority of respondents $(n=51,73.9 \%)$ were happy with the documentation stating either it was 'fine', 'ok', 'straightforward' or 'good'. Seven of those also specified that they used the documentation alongside the PGD as a checklist and found this approach useful. Pharmacists were asked what other 'common clinical conditions could be managed by the community pharmacists'. Of 44 responses $27.3 \%(n=12)$ stated 'none'. Of those who made a suggestion $(n=29)$, over half $(n=17, \quad 58.6 \%)$ mentioned skin conditions/ dermatology, particularly minor skin infection requiring flucloxacillin for example infected insect bites. Other conditions mentioned by more than one respondent were: chloramphenicol/acyclovir for shingles/naseptin/nystatin/fugal nail infections/ thrush for patients not on MAS/antiemetics for sickness. Single respondents suggested mefenamic acid/salbutamol/fexofenadine/NSAID.

Pharmacist participants were asked what difficulties or barriers they had encountered during the pharmacy first service. Responses $(n=62)$ were categorised. A third of respondents $(29 \%, n=18)$ had not had any difficulties. However, a third experienced problems with inappropriate referrals from GP practices $(29 \%, n=18)$. Time pressures were noted $(11.3 \%, n=7)$; some felt there was insufficient marketing or noted that the poster did not include exclusion criteria which caused problems $(9.7 \%, n=6)$. Training of locums was also noted $(4.8 \%, n=3)$.

\section{GP and GP reception staff feedback}

GPs from 34 of the 54 participating practices provided feedback (63\% response). The majority, but 
not all GPs were aware of the service and there was less awareness of the inclusion and exclusion criteria.

A summary of practice and GP responses is shown in Table 4. Reasons were sought on why the service was or was not useful. Of those who found the service useful to signpost patients to, many noted that it had a positive benefit on the practice workload as it freed up GP time and pressure on GP appointments.

Workload benefit for practice $(\mathrm{n}=9$ GPs and $\mathrm{n}=9$ reception staff)

'Great service - definitely takes workload off GP especially the UTI service which although a wee practice has really eased GP workload'

Patient convenience $(\mathrm{n}=2$ GPs $\mathrm{n}=4$ reception staff)

'Easier access for patients'

Quick access to treatment for patients $(\mathrm{n}=5)$

'Helps not pestering doctors - some patients not happy to be referred to pharmacy but most patients happy as dealt with quicker'

Other $(\mathrm{n}=11$ GPs and $\mathrm{n}=8$ reception staff)

'Pharmacy deal with most UTIs and send back to GP if complicated - most patients seen by pharmacy now and not $G P$ '

For those that did not find the service useful, this was either because they had no direct experience 'Only heard about it not used it in practice' or in one case because the GP was not supportive 'The Practice Manager stated GPs not keen on service- do not feel pharmacists qualified and said $H B$ do not advocate [??] Like to see their own patients' [reception staff].

GP and reception staff respondents were asked how the service might be improved. Responses identified the needs for clear, ongoing promotion of the service: 'Advertising for patients/ Posters for patient awareness'.

\section{Discussion}

\section{Summary of key findings}

Over a 5-month period 1189 cases were managed under the pharmacy first service, three quarters of which were for UTI. Of all cases, $77.9 \%$ were prescribed medication by the pharmacist, $9.1 \%$ were given advice only and $16.7 \%$ received referral to the GP practice (or out of hours). Objective clinical assessment of a sample of 30 referrals found all to be appropriate.

Feedback was received from 69 pharmacists, 34 GPs, 54 reception staff and 73 patients. Patients were very satisfied with the service, most frequently citing the quick and efficient access to treatment, and a professional service. Friendly, helpful and informative pharmacists were also cited frequently. Two thirds of GPs $(67 \%)$ who provided feedback and $59 \%$ of reception staff found the service useful. The main reason cited by both groups was it reduced pressure on GP appointments. Suggested improvements from pharmacists and GP staff were: improved documentation and feedback to GPs, more

Table 4 GP practice awareness and promotion of the service

\begin{tabular}{|c|c|c|c|}
\hline & Yes & No & Don't know \\
\hline & $n(\%)$ & $n(\%)$ & $n(\%)$ \\
\hline \multicolumn{4}{|l|}{$\mathrm{GPs}(n=34)$} \\
\hline Are you aware of the pharmacy first service? & $30(88.2)$ & $4(11.8)$ & - \\
\hline If yes, are you aware of the inclusion/exclusion criteria for the service? & $20(58.8)$ & $10(29.4)$ & - \\
\hline Do you advertise the pharmacy first service in the surgery? & $17(50.0)$ & $13(38.2)$ & 4 \\
\hline Do you find the pharmacy first service useful to signpost patient to? & $23(67.6)$ & $7(20.6)$ & 4 missing \\
\hline \multicolumn{4}{|l|}{ GP front desk staff $(n=54)$} \\
\hline Are you aware of the pharmacy first service? & $38(70.4)$ & 16 & - \\
\hline If yes, are you aware of the inclusion/exclusion criteria for the service? & $26(68.4)$ & $10(26.3)$ & - \\
\hline Do you advertise the pharmacy first service in the surgery? ${ }^{a}$ & $19(35.1)$ & $15(27.8)$ & $20(37.1)$ \\
\hline Do you find the pharmacy first service useful to signpost patient to? & $32(59.3)$ & $6(11.1)$ & 16 missing \\
\hline
\end{tabular}

a Twelve of the 19 advertised by poster, three had information displayed on the TV screen and two had information at the reception counter for reference. 
widespread marketing including posters with exclusion criteria listed and lists of participating pharmacies to be given to practice.

\section{Methodological considerations}

Patient feedback could be subject to response bias in which those who had a positive experience would be more likely to respond. Furthermore response was sought via pharmacies and patients were asked to return the questionnaire to the pharmacy. It is possible pharmacy staff may only have given questionnaires to those who had had a positive experience. For future evaluation it is suggested that patients are given a questionnaire and prepaid envelope so that responses can be returned directly to the evaluation team. Alternatively it might be possible to 'recruit' patients to provide feedback and an independent researcher might contact them directly by phone or email.

The reason for referral was often not reported. A referral form that included a GP assessment of the appropriateness of referral would be useful for further evaluation. This would both ensure the GP saw the referral and was made aware the patient had been referred and allow easier assessment of referrals. Inappropriate referrals could be identified more quickly although the sample of referrals randomly selected for assessment were all considered appropriate.

This evaluation only reviewed referrals to GPs and did not assess the appropriateness of treatment by pharmacists. A future evaluation might want to include this as there are potentially safety implications should pharmacists not follow the protocol. However in this project is was felt that the protocol was sufficient. Indeed it could be argued that this scheme is much 'safer' than existing practice such as the minor ailments scheme in which there is no protocol and pharmacists must apply their clinical judgement.

\section{Engagement and views of professionals}

Pharmacy participation was very high with 75 out of 76 pharmacies signing up to the service. This has subsequently increased to all 76 . Feedback from community pharmacy contractors was generally positive. There were clearly some early issues with study documentation which were subsequently revised. Although some pharmacists expressed their satisfaction in being involved in the service, others highlighted concerns about the time pressure in conducting consultations and completing documentation. This is a common theme in new service developments involving community pharmacy such as minor ailment schemes in Scotland (Paudyal et al., 2010) and New Medicine Service in England (Latif et al., 2016).

Several GPs and reception staff seemed unaware of the service, despite the service being operational for 5 months. There were clearly also problems with ensuring GPs were familiar with the inclusion and exclusion criteria so that they could direct patients appropriately. This had a knock-on effect for pharmacists and some patients as patients had to be referred back to the practice from the pharmacy. Reception staff noted that this could be avoided by them having a list of conditions and inclusion/exclusion criteria so they could direct patients appropriately. This information had been provided but had clearly not yet embedded in everyday practice. The introduction of the service provided an opportunity for community pharmacies and GP practices to improve their relationships. There was some evidence that one GP did not think this was an appropriate service for community pharmacy as they did not feel pharmacists was sufficient clinically trained. Others may have shared this view but this was not evident. This GP may be unaware of the training that participating pharmacists have undertaken and increased communication from the service might address this.

\section{Workload}

A major benefit noted by GP and reception staff was that the service freed up GP appointment times. In just 5 months, 1034 cases were managed in pharmacy rather than in general practice or OOHs. This represents a considerable shift in workload although it cannot be assumed that all of these cases would actually have attended either their GP or OOH. It is also possible that if such a service were expanded and promoted then patients might lower their threshold for using the service. Feedback was not sought from the $\mathrm{OOH}$ service as part of this evaluation but this would be desirable to allow any impact to be measured. Some GP reception staff had subjectively noticed the easing on GP appointments. The next step 
would be to undertake a simple cost benefit analysis, from an NHS and the patient perspective to fully quantify the costs and benefits of this service.

\section{Patient awareness and experience}

Patients were generally made aware of the service through the GP practice or the pharmacy. A number of GPs and pharmacists noted the need to increase marketing of the service. There is considerable scope to do this through other channels such as local radio, talks at community groups, etc. Furthermore, posters could be more widely distributed for example through community centres, libraries, etc. The poster was also criticised by some participants for not having sufficient information regarding inclusion and exclusion criteria.

The patient experience was very positive. The positive response was a consequence of quicker, more efficient access to treatment, and a professional service within the pharmacy. Pharmacists were considered informative and staff and pharmacists were perceived to be friendly and helpful. This was clearly valued as patients would have been in an unfamiliar situation as they may not have experienced a pharmacy consultation before. The introduction of services such as Pharmacy First help to inform the public of the skills pharmacists have and clinical role they can play in managing common clinical conditions. The positive view of patients was mirrored by comments from reception desk staff many of whom noted the benefit for patients. This positive feedback from patients has also been found in other initiates in which pharmacists have a developed role such as prescribing (Stewart et al., 2011; Tinelli et al., 2015). Similarly, quicker access to a professional service from pharmacists has been valued in other research as noted by a recent international review of pharmacist prescribing (Famiyeh, 2017).

\section{Future development of pharmacy first services}

The service worked well for UTI management with a high number of patients using the service. There was suggestion from pharmacists and reception staff that the age limit is too restrictive and increasing from 65 years to 70 or even 75 years should be considered. Impetigo also seemed an appropriate clinical area with few problems identified. However COPD treatment was more problematic. This was because patients were required to have hand-held cards documenting their treatment. However, many did not have these. The reasons for this requires further, focussed enquiry. There was a suggestion that some GPs did not want their COPD patients managed by community pharmacists. However, it might also be that the additional effort of issuing and then patients remembering the card might be problematic. The referrals made were considered appropriate. However, ongoing review of referrals would allow problems in the system to be identified earlier.

Regarding new clinical conditions, a considerable list was suggested with flucloxacillin for skin infections, chloramphenicol for conjunctivitis and aciclovir for shingles all mentioned. The suggestions made by pharmacists were informed by their experience of more common clinical conditions presenting in pharmacy that they have to refer to the GP because they cannot treat. In addition it would be useful to consider which of the conditions present most frequently in general practice and OOHs as freeing up appointment times is mutually beneficial for the patient and the GP practice/ OOHs. In Forth Valley the scheme has since been extended to include treatment of skin infections and mild inflammatory skin conditions, conjunctivitis and thrush. There is also a commitment from Scottish Government to increase access to community pharmacy as the first port of call for managing self-limiting illnesses in the new 'Achieving Excellence in Pharmaceutical Care - A Strategy for Scotland' (Scottish Government, 2017). Scottish Government have also allocated funding in 2017 to roll out the 'Pharmacy First' initiative based on this NHS Forth Valley work.

All professionals were asked for their views on how to improve the service. Both pharmacists and GPs noted the need for improved documentation to include feedback to GPs. GPs did actually get feedback which practice administrative staff will scan into the patient's Docman record, however they may not have been aware of this. As already highlighted more marketing was suggested to raise awareness. It was also felt that posters should list exclusion criteria. Training of regular pharmacy locums would further increase availability and reduce patients being passed between practice and pharmacy. 
The generally positive findings presented in this paper concur with wider, related literature on patient preference for the extended pharmacists prescribing role and their role in managing minor ailments. Discrete choice experiments to explore patient preference found that patients valued pharmacists in a prescribing role as an alternative to a doctor (Gerard et al., 2012). In the management of minor ailments in community pharmacy, the following attributes were identified as having the potential to increase uptake: a prompt service, trained staff who are friendly and approachable, and which helped patients understand and manage symptoms, and a local setting (Porteous et al., 2016).

In conclusion, Pharmacy First was well received by patients, pharmacists, GPs and reception staff. Some issues with awareness of inclusion/exclusion criteria and feedback systems to GP were highlighted but these can be addressed relatively easily through ongoing communication until the service becomes embedded into normal practice. It appears to work particularly well for UTIs and pharmacy referrals were clinically appropriate. There is some evidence that the service has relieved the pressure on GP appointments. However, a further evaluation to include a cost benefit evaluation would allow objective assessment of the value of this service. This would be of interest to other NHS areas considering introducing this service.

\section{Acknowledgements}

The authors would like to thank: Arlene Turnbull (NHS Forth Valley) for assisting with the training, roll out of the project and data collection, Catriona Matheson for undertaking the evaluation and assistance in the preparation of this manuscript, all the pharmacists, GPs and reception staff who were involved in implementing this services and who participated in the evaluation.

\section{Financial Support}

This research received no specific grant from any funding agency, commercial or not-for-profit sectors.

\section{Conflicts of Interest}

None.

\section{Ethical Standard}

The authors assert that all procedures contributing to this work comply with the ethical standards of the Royal Pharmaceutical Society and with the Helsinki Declaration of 1975, as revised in 2008.

\section{References}

Bissell, P., Savage, I. and Anderson, C. 2006: A qualitative study of pharmacists' perspectives on the supply of emergency hormonal contraception via patient group direction in the UK. Contraception 73, 265-70.

CWFI 2014: In depth review of the general practitioner workforce. Retrieved from www.cfwi.org.uk.

Famiyeh, I.M. and McCarthy, L. 2017: Pharmacist prescribing: a scoping review about the views and experiences of patients and the public. Research in Social and Administrative Pharmacy 13, 1-16.

Gerard, K., Tinelli, M., Latter, S., Blenkinsopp, A. and Smith, A. 2012: Valuing the extended role of prescribing pharmacist in general practice: results from a discrete choice experiment. Value in Health 15, 699-707.

Information Service Division (ISD) 2016: Primary Care Workforce Survey Scotland 2015: a survey of Scottish general practices and general practice out of hours services publica tion. Retrieved 18 January 2017 from http://www.isdscotland.org/Health-Topics/General-Practice/Publications/201606-14/2016-06-14-

PrimaryCareWorkforceSurveyScotland2015-Summary.pdf.

Joint Statement RPS (Scotland) RCGP (Scotland) 2011: Breaking down barriers: how pharmacists and general practitioners can work together to improve patient care. Retrieved 18 January 2017 from https://www.rpharms.com/ scottish-pharmacy-board-pdfs/breaking-down-the-barriersfinal-200120926.pdf.

Latif, A., Waring, J., Watmough, D., Barber, N., Chuter, A., Davies, J., Salema, N.E., Boyd, M.J. and Elliott, R.A. 2016: Examination of England's new medicine service (NMS) of complex health care interventions in community pharmacy. Research in Social Administrative Pharmacy 12, 966-89.

NICE 2013: Patient Group Directions: Medicines Practice Guideline (MPG2). Retrieved 12 December 2016 from https://www.nice.org.uk/guidance/mpg2.

Paudyal, V., Hansford, D., Cunningham, S. and Stewart, D. 2010: Cross-sectional survey of community pharmacists' views of the electronic minor ailment service in Scotland. International Journal of Pharmacy Practice 18, 194-201.

Porteous, T., Ryan, M., Bond, C., Watson, M. and Watson, V. 2016: Managing minor ailments; the public's preferences for attributes of community pharmacies. A discrete choice experiment. PLoS One 31, e0152257.

RPSGB 2015: Website statement. Retrieved 18 January 2017 from http://www.rpharms.com/our-campaigns/pharmacistsand-gp-surgeries.asp. 
Scottish Government 2017: Achieving excellence in pharmaceutical care launched August 2017. Retrieved 18 January 2017 from http://www.gov.scot/Publications/2017/08/4589/0.

Smith, J., Holder, H., Edwards, N., Jo Maybin, J., Parker, H., Rosen, R., Walsh, N. 2013: Securing the future of general practice: new models of primary care. London: The Kings Fund and Nuffield Trust.

Stewart, D.C., Maclure, K., Bond, C., Cunningham, S., Diack, L., George, J. and McCaig, D.J. 2011: Pharmacist prescribing in primary care: the views of patients across Great Britain who had experienced the service. International Journal of Pharmacy Practice 19, 328-32.

Tinelli, M., Blenkinsopp, A., Latter, S., Smith, A. and Chapman, S.R. 2015: Survey of patients' experiences and perceptions of care provided by nurse and pharmacist independent prescribers in primary care. Health Expectations $18,1241-255$. 